\title{
细菌纤维素基柔性锌离子电池正极的构筑及性能研究
}

\author{
张欣欣 ${ }^{a}$ 刘荣*, 王蕾*,a 付宏刚 $a$ \\ ( ${ }^{a}$ 黑龙江大学 功能无机材料化学教育部重点实验室 哈尔滨 150080) \\ ( $b$ 河北农业大学 海洋学院 秦皇岛 066000)
}

\begin{abstract}
摘要 柔性锌离子电池(ZIBs)具有高安全性、低成本和高能量密度等优势, 但是现有的 ZIBs 柔性电极难以兼具高电化 学性能和力学稳定性. 其中, 缺少适合的基底材料是限制柔性电极发展的关键. 本工作中, 以细菌纤维素(BC)为基底 材料, 结合原位聚合及真空过滤方法, 制备了具有 $3 \mathrm{D}$ 多孔结构的 $\mathrm{BC} /$ 聚苯胺/碳纳米管( $\mathrm{BC} / \mathrm{PANI} / \mathrm{CNTs})$ 柔性电极. BC 固有的高抗拉伸强度和超细纳米纤维网络结构等特点, 在赋予柔性电极高弯曲特性的同时, 还有利于活性物质的负载 及电解液离子的快速扩散. 结果表明, BC/PANI/CNTs 具有高柔韧性、 $7.3 \mathrm{mg} / \mathrm{cm}^{2}$ 的负载量和 $157 \mathrm{mAh} / \mathrm{g}$ 的比容量. 以 $\mathrm{BC} / \mathrm{PANI} / \mathrm{CNTs}$ 电极构建的准固态 ZIBs 展现了 $109 \mathrm{mAh} / \mathrm{g}$ 的比容量, 且 200 次充放电循环后容量保持率大于 $90 \%$. 关键词 锌离子电池; 柔性电极; 细菌纤维素; 聚苯胺; 碳纳米管
\end{abstract}

\section{Study on the Construction and Properties of Bacterial Cellulose-Based Cathode for Flexible Zn-Ion Batteries}

\author{
Xinxin Zhang ${ }^{a} \quad$ Rong Liu* ${ }^{*}$ Lei Wang*a Honggang $\mathrm{Fu}^{a}$ \\ ( ${ }^{a}$ Key Laboratory of Functional Inorganic Material Chemistry, Ministry of Education of the People's Republic of China, \\ Heilongjiang University, Harbin 150080, China) \\ ( ${ }^{b}$ Ocean College, Hebei Agricultural University, Qinhuangdao 066000, China)
}

\begin{abstract}
Flexible Zn-ion batteries (ZIBs) have been considered as desirable candidate of flexible energy storages due to its high safety, low cost and high energy density. However, there is a challenge that ZIBs can possess both high electrochemistry properties and good mechanical stability. Among them, the lack of suitable substrate material has long been the major obstacles against the development of flexible electrode for ZIBs. In this work, we prepared the bacterial cellulose/polyaniline/ carbon nanotubes (BC/PANI/CNTs) flexible electrode through a simple in-situ polymerization with subsequent vacuum filtration. Typically, the $\mathrm{BC}$ suspension and aniline monomer were dissolved into $\mathrm{HCl}$ solution. After the ammonium persulfate dispersed in another $\mathrm{HCl}$ aqueous solution, the obtained solution was subsequently added dropwise into the above mixture in an ice bath. Then, the acid treated CNTs was added to achieve the whole dispersion. The resulted film was collected by vacuum filtration and the BC/PANI/CNTs flexible electrode was finally obtained by further dried in an oven. Benefiting from the $\mathrm{BC}$ substrate, the $\mathrm{BC} / \mathrm{PANI} / \mathrm{CNTs}$ flexible electrode possesses a 3D porous structure, which can provide the rapid diffusion channel of electrolyte ion and improve the contact between the active material and electrolyte ion. Moreover, the BC substrate endow the BC/PANI/CNTs electrode with high flexibility and large mass loading of active materials. The scanning electron microscopy (SEM) image of the BC/PANI/CNTs electrode shows that the PANI is uniformly anchored on the surface of $\mathrm{BC}$ nanofiber, and the CNTs is dispersed in the porous network and connected with BC/PANI nanofibers. Furthermore, the X-ray diffraction (XRD), Raman spectra, Fourier transform infrared spectra (FT-IR) and X-ray photoelectron spectroscopy (XPS) confirm the structure of the BC/PANI/CNTs. The BC/PANI/CNTs, tested as a flexible cathode for ZIBs, achieve both good electrochemical performance, such as mass loading of $7.3 \mathrm{mg} / \mathrm{cm}^{2}$, gravimetric capacity of $157 \mathrm{mAh} / \mathrm{g}$, areal capacity of $1.148 \mathrm{mAh} / \mathrm{cm}^{2}$, and excellent mechanical flexibility, which can be bended, twisted and rolled. To further explore the application value of this flexible electrode in electronic equipment, the quasi-solid-state ZIBs was prepared with the BC/PANI/CNTs as cathode, the $\mathrm{Zn} /$ carbon cloth (CC) as anode, and the $\mathrm{ZnSO}_{4} /$ polyvinyl alcohol (PVA) as gel electrolyte, respectively. The assembled quasi-solid-state ZIBs deliver a specific capacity of $109 \mathrm{mAh} / \mathrm{g}$, and over $90 \%$ of the initial capacity is retained after 200 charge/discharge cycles at a low current density of $0.5 \mathrm{~mA} / \mathrm{cm}^{2}$. The low-cost BC/PANI/CNTs flexible electrode has excellent performance and mechanical property, providing a feasible scheme for the scalable application of ZIBs.
\end{abstract}

Keywords Zn-ion battery; flexible electrode; bacterial cellulose; polyaniline; CNTs

\footnotetext{
*E-mail: 562204852@qq.com; wanglei0525@hlju.edu.cn; Tel.: 0451-86604330

Received January 31, 2021; published March 29, 2021.

Supporting information for this article is available free of charge via the Internet at http://sioc-journal.cn.

Project supported by the National Key R\&D Program of China (2018YFE0201704), the National Natural Science Foundation of China (21771059, 21631004) and the Natural Science Foundation of Heilongiiang Province (YQ2019B007).

项目受国家重点研发计划(2018YFE0201704)、国家自然科学基金(21771059,21631004)和黑龙江省自然科学基金(YQ2019B007)资助.
} 


\section{1 引言}

随着可穿戴及便携式电子设备的快速发展, 人们对 高性能柔性储能设备的需求日益增长 ${ }^{[1-2]}$. 近年来, 锌离 子电池(ZIBs)作为一种新型的电化学储能器件, 因其锌 负极材料资源丰富、成本低廉、理论比容量高 $(820$ $\mathrm{mAh} / \mathrm{g})$ 、氧化还原电位低 $(-0.76 \mathrm{~V}$ vs. SHE), 并可采用 无毒安全的水系电解液, 被认为是理想的柔性储能装置 之一[-5]. 在高性能柔性 ZIBs 中, 柔性电极的设计和制 备无疑处于核心位置. 然而, 如何制备兼具高电化学性 能和力学稳定性的柔性电极, 一直是 ZIBs 面临的挑战. 究其原因, 在于目前的 ZIBs 柔性电极所采用的基底材 料难以在保证高柔韧性的同时提供高的比表面积, 导致 活性物质负载量不足 ${ }^{[6-9]}$. 因此, 开发合适的基底材料是 推动柔性 ZIBs 发展的关键所在.

细菌纤维素 $(\mathrm{BC})$ 是利用微生物发酵而产生的一种 可再生生物材料. $\mathrm{BC}$ 固有的特殊微观结构和物理/化学 特性, 为其在水系能源存储的应用提供了广阔的前景. $\mathrm{BC}$ 具有由互相交错的纳米纤维构成的 $3 \mathrm{D}$ 网络结构, 可 提供更高的比表面积, 为活性物质形成大量负载提供了 条件. $\mathrm{BC}$ 表面存在着大量的羟基官能团, 可通过氢键和 静电引力等作用与活性物质形成紧密结合 ${ }^{[10-17]}$, 同时, 大量亲水性基团的存在使得 $\mathrm{BC}$ 具有高持水性, 这有利 于在水系储能设备中的应用. 此外, $\mathrm{BC}$ 的高抗拉强度有 利于柔性电极力学性能的提高. 然而, 极少有关于 $\mathrm{BC}$ 基底的 ZIBs 柔性电极的文献报道. 因此, 我们有必要采 用 $\mathrm{BC}$ 来负载活性材料, 用以进一步提升柔性 ZIBs 的性 能. 众所周知, 聚苯胺(PANI) 是一种很有前途的活性电 极材料, 其具有比容量高、稳定性好、成本低和易于制 备等优点 ${ }^{[18-22]}$. 与传统的无机材料相比, PANI 的储能机 理完全不同 ${ }^{[23]}$. PANI 中的氮 $(\mathrm{N})$ 存在未掺杂态 $(=\mathrm{N}-)$ 和 掺杂态 $\left(=\mathrm{NH}^{+}-\right)$两种形态. 在放电过程中, PANI 中的 $=\mathrm{N}$ 一获得电子被还原为 $-\mathrm{N}^{-}$- 形态, 并与电解液中的 $\mathrm{Zn}^{2+}$ 相互反应. 同时, PANI 中的 $=\mathrm{NH}^{+}$-转化为 $-\mathrm{NH}-$, 而与之作用的阴离子从 PANI 中脱出. 在充电 过程中, PANI 中的 $-\mathrm{N}^{-}$- 被氧化为 $=\mathrm{N}-$, 与 $-\mathrm{N}^{-}-$相 互作用的 $\mathrm{Zn}^{2+}$ 从 PANI 中脱出, 与此同时, PANI 中的 $-\mathrm{NH}-$ 转化为 $=\mathrm{NH}^{+}$-, 而电解液中的阴离子吸附于 活性位点, 因此, PANI//Zn 属于典型的双离子电池.

此外, 近年来的研究表明, 在 PANI 材料中引入高 导电碳材料可以提高 PANI 的电子导电性 ${ }^{[24]}$, 由此得到 更高容量的复合电极材料. 在众多导电碳材料中, 碳纳 米管 $(\mathrm{CNTS})$ 具有一维(1D) 结构, 因此不仅提供了沿 CNTs 方向的连续导电路径还展现出更好的柔韧性 ${ }^{[25-26]}$.

基于以上分析, 我们以 $\mathrm{BC}$ 为基底材料, 通过简单 的原位氧化聚合及真空过滤技术, 制备了具有 3D 网络 结构的 BC/PANI/CNTs 柔性电极. 相对于未添加 BC 的 PANI/CNTs 材料, 该柔性电极不仅展现出突出的柔韧 性, 同时具有开放多孔的电极结构, 为离子的快速传输
提供了通道，提高了活性材料利用率. 因此，利用 $\mathrm{BC}$ 、 PANI 和 CNTs 之间的有效协同作用与界面相互作用, $\mathrm{BC} / \mathrm{PANI} / \mathrm{CNTs}$ 同时获得了高电化学性能和力学稳定 性. 所制备的基于 $\mathrm{BC} / \mathrm{PANI} / \mathrm{CNTs}$ 柔性电极的 ZIBs 在 1 $\mathrm{mA} / \mathrm{cm}^{2}$ 电流密度时, 其放电比容量可达 $157 \mathrm{mAh} / \mathrm{g}$ $\left(1.148 \mathrm{mAh} / \mathrm{cm}^{2}\right)$, 为 PANI/CNTs 材料的 1.3 倍. 进一步 将 $\mathrm{BC} / \mathrm{PANI} / \mathrm{CNTs}$ 正极, $\mathrm{Zn} / \mathrm{CC}$ 负极和 $\mathrm{PVA} / \mathrm{ZnSO}_{4}$ 凝胶 电解质组装准固态柔性 ZIBs. 该 ZIBs 在 $0.5 \mathrm{~mA} / \mathrm{cm}^{2}$ 的 电流密度下进行 200 次充放电循环后仍可以保持 $90 \%$ 以 上的容量. BC/PANI/CNTs 作为一种低成本方法制备的 正极材料, 却拥有优异的电化学性能, 为 ZIBs 未来的规 模化开发提供了一种可行的方案.

\section{2 结果与讨论}

\section{1 柔性电极的结构表征}

BC/PANI/CNTs 柔性电极的制备流程如图 1A 所示. 我们首先制备 $\mathrm{BC}$ 悬浮液, 然后在适量 $\mathrm{BC}$ 悬浮液中分 别加入苯胺单体 $(\mathrm{AN})$ 、过硫酸铵 $(\mathrm{APS})$ 、盐酸 $(\mathrm{HCl})$, 之 后在低温条件下原位聚合使得 PANI 均匀分布在 BC 纤 维表面, 合成了 $\mathrm{BC} / \mathrm{PANI}$. 最后, 在 $\mathrm{BC} / \mathrm{PANI}$ 混合液中 加入 CNTs, 并采用真空过滤技术, 制备了 $\mathrm{BC} / \mathrm{PANI} / \mathrm{CNTS}$ 柔性自支撑电极.

图 $1 \mathrm{~B} \sim 1 \mathrm{G}$ 分别为 $\mathrm{BC} 、 \mathrm{BC} / \mathrm{PANI}$ 和 $\mathrm{BC} / \mathrm{PANI} / \mathrm{CNTs}$ 样品的扫描电子显微镜 $(\mathrm{SEM})$ 照片. 从图 $1 \mathrm{~B}, 1 \mathrm{C}$ 中可以 清晰地看出, BC 由直径在 10 100 $\mathrm{nm}$ 之间的超细纤维 构成, 并且各纤维相互交错形成了网络结构. 显然, 经 过原位聚合后, BC 纤维表面均匀贴合了一层 PANI 导电 聚合物(图 1D). 高倍率 SEM 图像(图 1E)显示, PANI 呈 现出棒状的微观形貌, 并且只有微量的 PANI 散落在纤 维之间的空隙中, 验证了 $\mathrm{AN}$ 在 $\mathrm{BC}$ 纤维表面的有效聚 合. 进一步在 BC/PANI 材料中引入 CNTs, 如图 $1 \mathrm{~F}, 1 \mathrm{G}$ 所示, $\mathrm{CNTs}$ 较均匀搭接在 $\mathrm{BC} / \mathrm{PANI}$ 纤维上, 并相互连接 穿插于各 BC/PANI 间，在 BC/PANI/CNTs 柔性电极中构 建了 $3 \mathrm{D}$ 导电网络, 为电子的快速传输提供了保障. 同 时, CNTs 的引入并没有改变 BC/PANI 材料原有的多孔 结构, 这为电解液离子的扩散提供了通道, 有助于电解 液离子向活性物质表面快速扩散, 增加了有效活性位 点. 相对于 BC/PANI/CNTs, 在 PANI/CNTs 样品的 SEM 图像中(图 S1)可以观察到 PANI 的团聚, 并且没有形成 有效的 $3 \mathrm{D}$ 多孔网络结构. 为了验证 $\mathrm{BC}$ 的引入可以提供 亲水性, 我们对 $\mathrm{BC}, \mathrm{BC} / \mathrm{PANI}, \mathrm{PANI} / \mathrm{CNT}$ 和 BC/PANI/ $\mathrm{CNTs}$ 三个样品的接触角进行了测试. 如图 $\mathrm{S} 2$ 所示, $\mathrm{BC}$ 膜是高度亲水的, 水滴在其表面迅速铺展, 接触角仅为 $16.4^{\circ}$. BC/PANI 和 BC/PANI/CNTs 膜的接触角分别为 $39.1^{\circ}$ 和 $69.7^{\circ}$, 而未加 $\mathrm{BC}$ 的 PANI/CNTs 接触角为 $79.4^{\circ}$, 表明 $\mathrm{BC}$ 的引入使得膜材料的亲水性得到提升, 这有利 于在水系储能设备中的应用. 


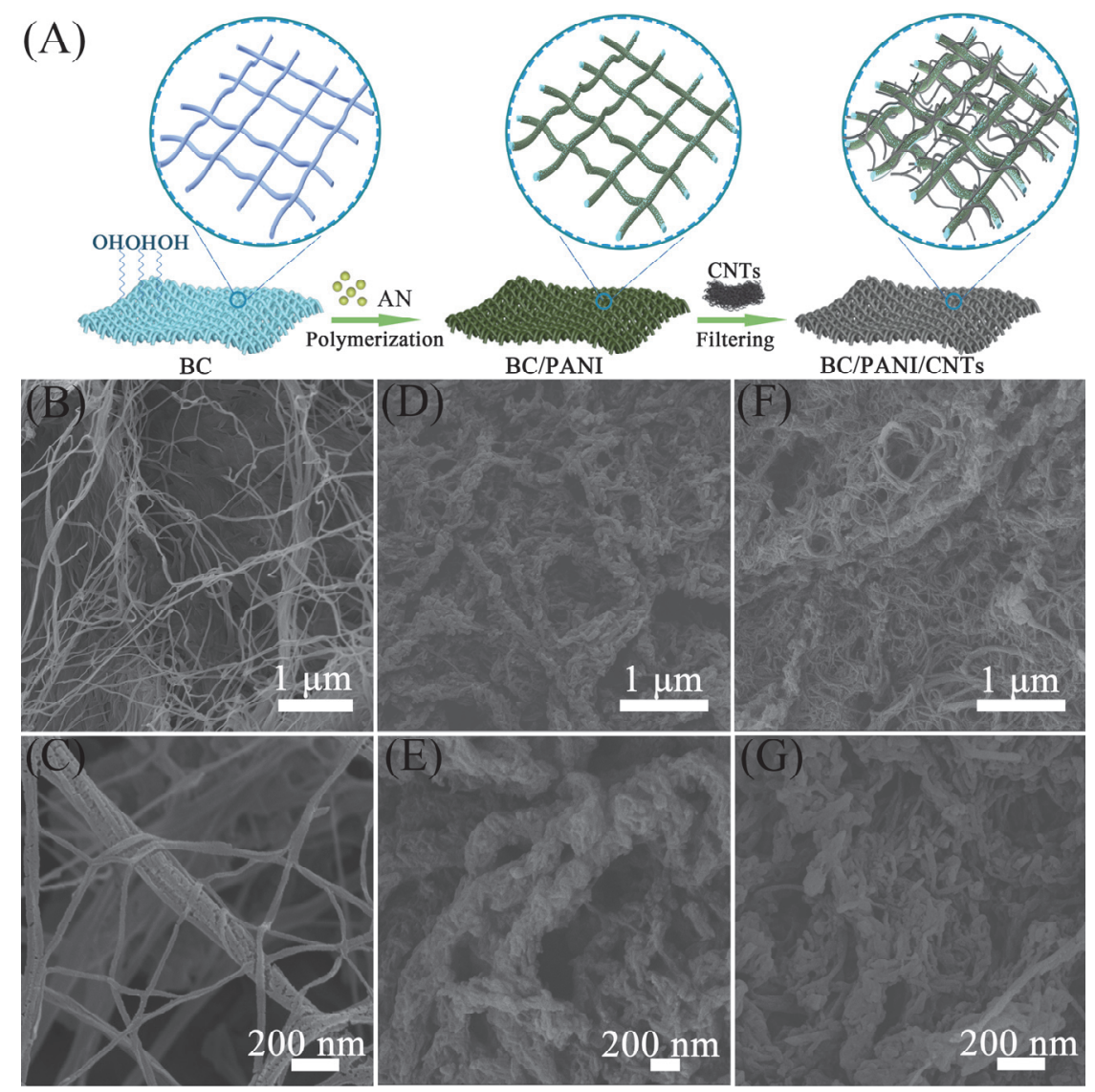

图 1 (A) BC/PANI/CNTs 柔性电极的制备流程图; (B, C) BC 的 SEM 照片; (D, E) BC/PANI 的 SEM 照片; (F, G) BC/PANI/CNTs 的 SEM 照片 Figure 1 (A) Schematic of the fabrication process of BC/PANI/CNTs flexible electrode; (B, C) SEM images of BC; (D, E) SEM images of BC/PANI; $(\mathrm{F}, \mathrm{G})$ SEM images of BC/PANI/CNTs

因此, BC/PANI/CNTs 电极同时展现出优异的电子 和离子导电性，其可作为自支撑电极而无需其它集流 体.

为了分析材料的晶体结构, 我们对 $\mathrm{BC} 、 \mathrm{BC} / \mathrm{PANI}$ 和 BC/PANI/CNTs 样品进行了 X射线衍射(XRD)表征(图 $2 \mathrm{~A})$. 由 $\mathrm{BC}$ 的 XRD 图谱可以看出, 衍射角 $2 \theta$ 在 $14.2^{\circ}$ 、 $16.7^{\circ}$ 和 $22.3^{\circ}$ 位置出现了对应于(1-10)、(110)和(020)衍 射面的特征峰 ${ }^{[27-28]}$. 在 BC/PANI 的 XRD 图谱中, 仅在 $25.0^{\circ}$ 处出现了较弱的新特征峰, 对应于 PANI 的(002)衍 射面, 这主要是因为相对于高结晶度的 BC, PANI 的结 晶度较低 ${ }^{[29-30]}$. 在 BC/PANI/CNTs 样品的 XRD 图谱中, 衍射角 $2 \theta$ 在 $25.9^{\circ}$ 和 $42.9^{\circ}$ 位置可归属于 CNTs 的特征衍 射峰, 这证明了 CNTs 成功的引入 $\mathrm{BC} / \mathrm{PANI}$ 中[31-32]. 图 $2 \mathrm{~B}$ 是 $\mathrm{BC} 、 \mathrm{BC} / \mathrm{PANI}$ 和 $\mathrm{BC} / \mathrm{PANI} / \mathrm{CNTs}$ 样品的傅里叶红 外(FT-IR)光谱图. 在 $\mathrm{BC}$ 的 FT-IR 谱图中, 位于 1030 $\mathrm{cm}^{-1}$ 的峰归因于 $\mathrm{C}-\mathrm{O}-\mathrm{C}$ 的骨架振动; $1315 \mathrm{~cm}^{-1}$ 处的 峰归因于 $\mathrm{O}-\mathrm{H}$ 的弯曲振动; $1162 \mathrm{~cm}^{-1}$ 位置的峰对应于 $\mathrm{C}-\mathrm{O}$ 的拉伸振动; $1642 \mathrm{~cm}^{-1}$ 和 $2895 \mathrm{~cm}^{-1}$ 位置的峰分 别表示吸收水的 $\mathrm{O}-\mathrm{H}$ 弯曲振动和 $\mathrm{C}-\mathrm{H}$ 拉伸振动; $3341 \mathrm{~cm}^{-1}$ 处的特征峰对应于 $\mathrm{O}-\mathrm{H}$ 的拉伸振动 ${ }^{[33-34]}$. 在 PANI 的 FT-IR 谱图中, 位于 $1471 \mathrm{~cm}^{-1}$ 和 $1558 \mathrm{~cm}^{-1}$ 处 的峰分别归属于醌和苯环中的 $\mathrm{C}=\mathrm{C}$ 拉伸振动 ${ }^{[35-36]}$. 显
然, PANI 在 BC 纤维聚合后制备的 BC/PANI 包含了 PANI 和 BC 的主要特征峰. 从 BC/PANI/CNTs 的 FT-IR 光谱可以看出, 进一步引入的 $\mathrm{CNTs}$, 并没有引起 BC/PANI FT-IR 光谱的显著变化, 除峰位微量偏移, 归 属于 BC 和 PANI 的特征峰仍清晰可见. 图 S3 是 BC/PANI/CNTs 的拉曼 (Raman)光谱. 如图所示, 位于 $1163 \mathrm{~cm}^{-1}$ 和 $1487 \mathrm{~cm}^{-1}$ 的特征峰归属于为 PANI 醌环的 $\mathrm{C}-\mathrm{H}$ 弯曲和 $\mathrm{C}-\mathrm{C}$ 振动, 而 $1349 \mathrm{~cm}^{-1}$ 和 $1588 \mathrm{~cm}^{-1}$ 分 别对应于 CNTs 的 D 峰和 $G$ 峰, 进一步证明了

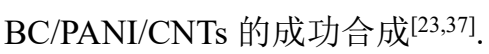

我们进一步通过 X 射线光电子能谱(XPS)分析样品 的组成. 图 S4 是 BC/PANI/CNTs 样品的 XPS 全谱. 如 图所示, BC/PANI/CNTs 主要由 $\mathrm{C} 、 \mathrm{~N}$ 和 $\mathrm{O}$ 三种元素构 成, 而其中的 $\mathrm{N}$ 元素来自于活性物质 PANI. 图 $2 \mathrm{C}$ 是 $\mathrm{BC} / \mathrm{PANI} / \mathrm{CNTs}$ 样品的高分辨率 C1s XPS 谱图, 通过拟 合可以分为位于 284.6、285.5、286.5 和 $288.0 \mathrm{eV}$ 处的 四个峰, 分别对应于 $\mathrm{C}-\mathrm{C} / \mathrm{C}=\mathrm{C} 、 \mathrm{C}-\mathrm{N} 、 \mathrm{C}-\mathrm{O}$ 和 $\mathrm{C}=$ $\mathrm{O} / \mathrm{O}-\mathrm{C}=\mathrm{O}^{[38-39]}$. 图 $2 \mathrm{D}$ 是高分辨率 $\mathrm{N} 1 \mathrm{~s}$ XPS 谱图, 其 主要由位于 398.6、399.6 和 $401.3 \mathrm{eV}$ 处的三个峰构成, 分别对应于 $=\mathrm{N}-、-\mathrm{NH}-、=\mathrm{N}^{+} \mathrm{H}-$ 三种键合形 式 ${ }^{[40-41]}$. 图 3 展现了 $\mathrm{BC} / \mathrm{PANI} / \mathrm{CNTs}$ 柔性电极的力学特 性. 相比于 BC 膜(图 S5), BC/PANI/CNTs 柔性电极的表 
观颜色明显不同, 呈现出深黑色(图 3A). 得益于 $\mathrm{BC}$ 的 特殊微观网络结构及高力学强度, BC/PANI/CNTs 柔性 电极具有良好的柔韧性, 在弯曲、折叠状态下, 该电极 未出现任何损坏的现象 (图 3B 3D). 而未添加 BC 的 PANI/CNTs 呈粉末态, 无法作为自支撑柔性电极使用. PANI/CNTs 需要采用传统的刮涂工艺, 即将 PANI/CNTs 粉末与粘结剂混合后涂覆在集流体上, 而不导电的粘结
剂不仅增加了 ZIBs 的电阻，同时增加了 ZIBs 的成本， 不利于 ZIBs 的实际应用. 相反, BC/PANI/CNTs 柔性电 极, 在 $7.30 \mathrm{mg} / \mathrm{cm}^{2}$ 的高活性物质负载量时经数百次重 复弯曲后仍表现出很高的柔韧性. 结果表明, BC 作为优 异的柔性基底，在 ZIBs 柔性电极的制备中展现了巨大 的潜力.
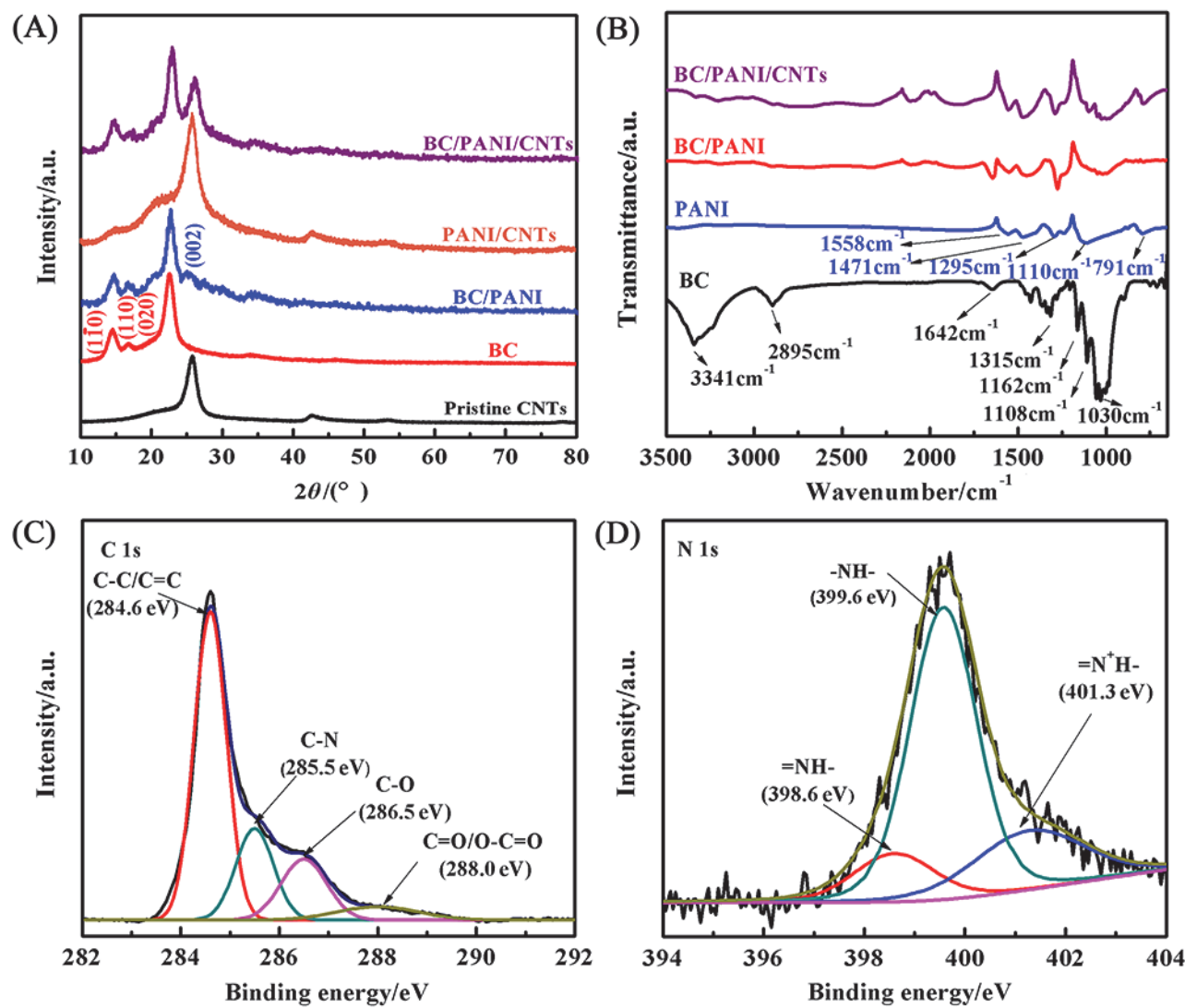

图 2 (A) BC、BC/PANI 和 BC/PANI/CNTs 样品的 XRD 图谱; (B) BC、BC/PANI 和 BC/PANI/CNTs 样品的 FT-IR 谱图; (C) BC/PANI/CNTs 的高分 辨 $\mathrm{C} 1 \mathrm{~s}$ 谱图和(D) $\mathrm{N} 1 \mathrm{~s}$ 谱图

Figure 2 (A) XRD patterns of $\mathrm{BC}, \mathrm{BC} / \mathrm{PANI}$ and $\mathrm{BC} / \mathrm{PANI} / \mathrm{CNTs}$ samples; (B) FT-IR spectra of BC, BC/PANI and BC/PANI/CNTs samples; high-resolution (C) C1s and (D) N1s XPS spectra of BC/PANI/CNTs sample
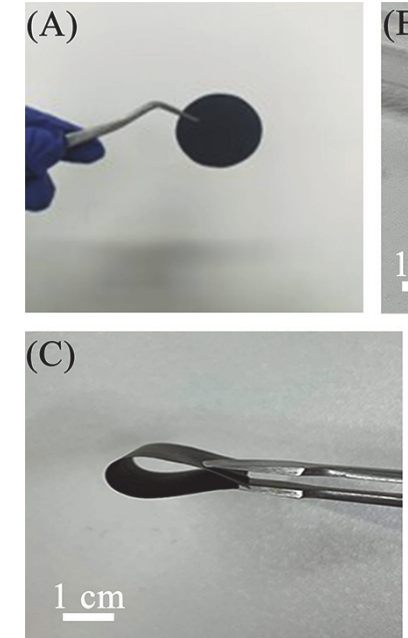
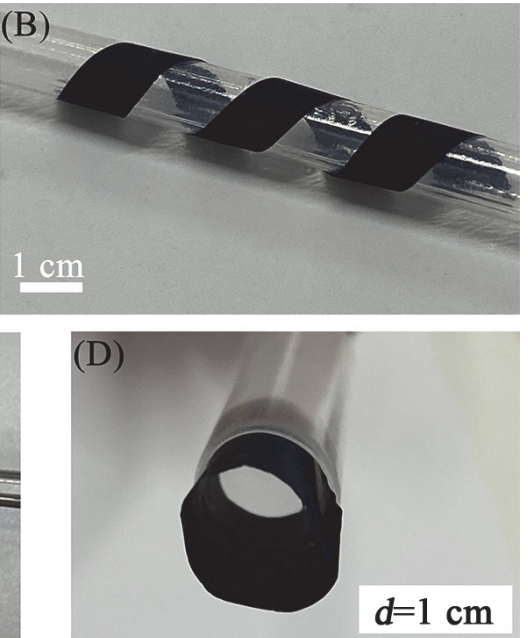

图 $3 \mathrm{BC} / \mathrm{PANI} / \mathrm{CNTs}$ 柔性电极处于(A)自然状态、(B)卷曲状态和 $(\mathrm{C}, \mathrm{D})$ 弯曲状态下的光学照片

Figure 3 Optical images of the BC/PANI/CNTs flexible electrode when they are in the (A) natural, (B) rolled, and (C, D) bended state 


\section{2 柔性电极的电化学性能研究}

我们通过测试 BC/PANI/CNTs 、 BC/PANI 和 PANI/CNTs 电极在 $2 \mathrm{~mol} / \mathrm{L} \mathrm{ZnSO}_{4}$ 电解液中的循环伏安 (CV) 和充放电特性来分析各样品的电化学行为. 图 $4 \mathrm{~A}$ 为基于 $\mathrm{BC} / \mathrm{PANI} / \mathrm{CNTs}$ 电极的 ZIBs 装置示意图. 从图 $\mathrm{S} 6$ 可以看出, 在电流密度为 $1 \mathrm{~mA} / \mathrm{cm}^{2}$ 时, 未添加 $\mathrm{BC}$ 的 PANI/CNTs 电极 $\left(7.30 \mathrm{mg} / \mathrm{cm}^{2}\right)$ 具有 $119 \mathrm{mAh} / \mathrm{g}(0.871$ $\left.\mathrm{mAh} / \mathrm{cm}^{2}\right)$ 的比容量. 图 $\mathrm{S} 7$ 为不同 $\mathrm{AN}$ 添加量 $(0.06 、 0.07$ 和 $0.08 \mathrm{~mL}$ )制备的复合电极在 $1 \mathrm{~mA} / \mathrm{cm}^{2}$ 电流密度时的 比容量图, 图 S8 和 S9 分别是不同电流密度下的比容量 和倍率性能图. 结果表明, BC/PANI(或 BC/PANI-0.07) 不仅具有 $0.811 \mathrm{mAh} / \mathrm{cm}^{2}$ 高的面积比容量和突出的倍率 性能, 同时在所有电极中展现出最高的质量比容量 (105.46 mAh/g,), 基于以上分析, 我们在 $\mathrm{BC} / \mathrm{PANI}$ 中引
入 CNTs. 如图 4B 所示, BC/PANI/CNTs 柔性电极在 1 $\mathrm{mA} / \mathrm{cm}^{2}$ 时展现出 $157 \mathrm{mAh} / \mathrm{g}\left(1.148 \mathrm{mAh} / \mathrm{cm}^{2}\right)$ 的比容量, 明显高于 BC/PANI 和 PANI/CNTs. 此外, 当电流密度从 $1 \mathrm{~mA} / \mathrm{cm}^{2}$ 提高至 $25 \mathrm{~mA} / \mathrm{cm}^{2}$ 时, BC/PANI/CNTs 在 0.5 $1.6 \mathrm{~V}$ 的电位区间内仍保持相似的充放电曲线形态(图 $4 \mathrm{C})$, 表明 $\mathrm{BC} / \mathrm{PANI} / \mathrm{CNTs}$ 柔性电极具有较好的倍率性 能.

图 4D 是 BC/PANI/CNTs、BC/PANI 和 PANI/CNTs 电极的倍率性能对比图. 当电流密度为 $15 \mathrm{~mA} / \mathrm{cm}^{2}$ 时, $\mathrm{BC} / \mathrm{PANI} / \mathrm{CNTs}$ 电极的容量保持率为 $71 \%$ ，高于 BC/PANI 的 58\%和 PANI/CNTs 的 $63 \%$. 我们进一步通 过对比各电极的电化学阻抗谱图 (EIS) 来分析 $\mathrm{BC} / \mathrm{PANI} / \mathrm{CNTs}$ 倍率性能高的原因. 如图 4E 所示, 所得 到的 EIS 均由高频区域的半圆和低频区域的斜线组成,
(A)

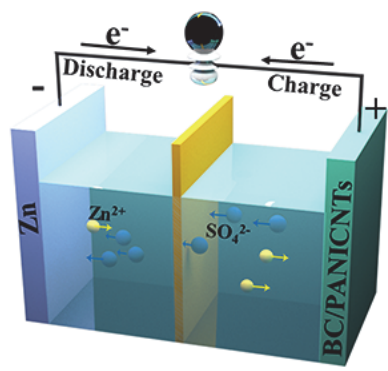

(B)

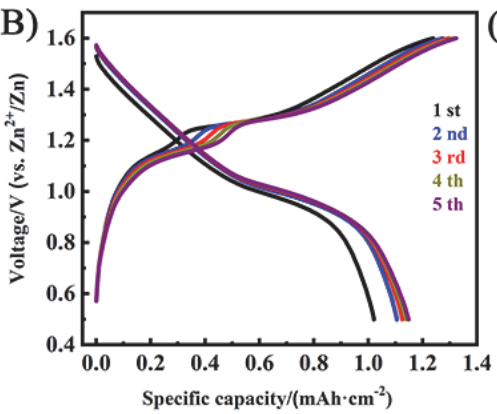

(D)

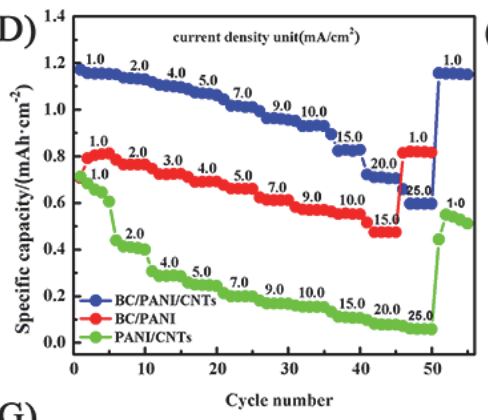

(G)

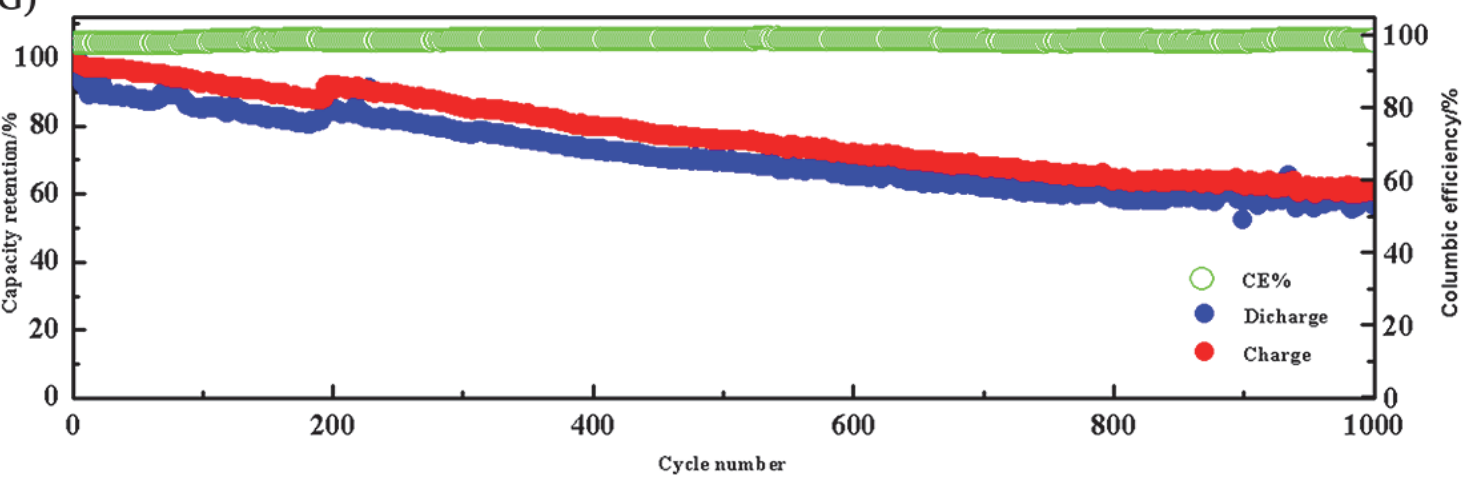

图 4 (A) ZIBs 装置示意图; (B) BC/PANI/CNTs 在 $1 \mathrm{~mA} / \mathrm{cm}^{2}$ 电流密度下的充放电曲线; (C) BC/PANI/CNTs 在不同电流密度下对应的充放电比容量; (D) BC/PANI, PANI/CNTs 和 BC/PANI/CNTs 在不同电流密度下的比容量对比图; (E) BC/PANI, PANI/CNTs 和 BC/PANI/CNTs 的电化学阻抗图和等 效电路图; (F) BC/PANI/CNTs 在 $0.1 \mathrm{mV} / \mathrm{s}$ 扫描速度下的 $\mathrm{CV}$ 曲线; (G) BC/PANI/CNTs 在 $10 \mathrm{~mA} / \mathrm{cm}^{2}$ 电流密度下经 1000 次充放电循环的容量保持 率曲线

Figure 4 (A) Schematic of the ZIBs device; (B) galvanostatic charge-discharge curves of BC/PANI/CNTs at $1 \mathrm{~mA} / \mathrm{cm}^{2}$; (C) charge-discharge profiles of $\mathrm{BC} / \mathrm{PANI} / \mathrm{CNTs}$ at different current densities; (D) rate performance of BC/PANI, PANI/CNTs and BC/PANI/CNTs, at various current densities; (E) EIS plots and the equivalent circuit of BC/PANI, PANI/CNTs and BC/PANI/CNTs; (F) cyclic voltammetry curve of BC/PANI/CNTs at a scan rate of 0.1 $\mathrm{mV} / \mathrm{s} ;(\mathrm{G})$ cycling performance of BC/PANI/CNTs at a current density of $10 \mathrm{~mA} / \mathrm{cm}^{2}$ 
其中, 高频区域的半圆表示传荷电阻 $\left(R_{\mathrm{ct}}\right)$. 拟合结果表 明, $\mathrm{BC} / \mathrm{PANI} / \mathrm{CNTs}$ 的串联电阻 $\left(R_{\mathrm{s}}\right)$ 值为 $1.486 \Omega, R_{\mathrm{ct}}$ 值仅 为 $14.37 \Omega$, 而 PANI/CNTs 和 BC/PANI 的 $R_{\mathrm{s}}$ 值为 2.387 和 $2.618 \Omega, R_{\mathrm{ct}}$ 值分别达到了 $62.12 \Omega$ 和 $71.72 \Omega$. 因此, 在复合电极中 BC、 PANI 及 CNTs 之间可以起到协同作 用, 明显提高了电荷传输动力.

我们进一步探究了不同 CNTs 负载量对 ZIBs 电化 学性能的影响. 图 $\mathrm{S} 10 \sim \mathrm{S} 12$ 分别是 $\mathrm{CNTs}$ 为 5、10 和 $15 \mathrm{mg}$ 时在 $1 \mathrm{~mA} / \mathrm{cm}^{2}$ 下的充放电曲线, 不同电流密度下 的充放电曲线和倍率性能图. 结果表明, CNTs 的含量对 $\mathrm{BC} / \mathrm{PANI} / \mathrm{CNTs}$ 电极的电化学性能有影响, 而 $\mathrm{BC} / \mathrm{PANI} / \mathrm{CNTs}$ (或 BC/PANI/CNTs-10)的性能为最佳. 值 得注意的是, 目前文献报道的 $\mathrm{ZIBs}$ 柔性正极活性物质 负载量在 $2 \mathrm{mg} / \mathrm{cm}^{2}$ 左右, 而 $\mathrm{BC} / \mathrm{PANI} / \mathrm{CNTs}$ 获得了约 $7.30 \mathrm{mg} / \mathrm{cm}^{2}$ 的高负载量, 同时展现出高比容量. 图 $4 \mathrm{~F}$ 是 BC/PANI/CNTs 在 $0.1 \mathrm{mV} / \mathrm{s}$ 的扫描速率下的 $\mathrm{CV}$ 曲线. 如图所示, CV 曲线在 1.20/0.89 和 1.29/0.99 V 处出现了 两对氧化还原峰, 这主要是由活性材料 PANI 在半导体 态和导电态之间的转换引起的. 此外, 循环性能是评价 ZIBs 的实际应用的重要指标. 如图 4G 所示, ZIBs 在电 流密度为 $10 \mathrm{~mA} / \mathrm{cm}^{2}$ 时, 经过 1000 次充放电循环后, 容 量仍然保持 $50 \%$ 以上, 库仑效率在整个循环寿命内接近 $100 \%$. 因此, 基于 BC/PANI/CNTs 柔性电极的 ZIBs 具有 显著的循环稳定性.

为了进一步探讨 BC/PANI/CNTs 柔性电极在电子设 备中的应用价值, 我们以该电极为正极, 以 $\mathrm{Zn} / \mathrm{CC}$ 为负 极, 以 $\mathrm{ZnSO}_{4} / \mathrm{PVA}$ 为凝胶电解质, 装配准固态 $\mathrm{ZIBs}$. 选 择 $\mathrm{Zn} / \mathrm{CC}$ 作为负极是由于传统的 $\mathrm{Zn}$ 䇴柔韧性不足, 在 机械形变过程中容易出现折损, 无法满足高性能柔性
ZIBs 的需求. 因此, 我们采用电化学沉积法, 将金属 $\mathrm{Zn}$ 负载在力学性能更佳的 $\mathrm{CC}$ 上. 同时, $\mathrm{Zn} / \mathrm{CC}$ 的设计还有 利于电化学性能的改善. 一方面, 沉积的纳米 $\mathrm{Zn}$ 可以 减缓充放电过程中 $\mathrm{Zn}$ 枝晶的产生, 提高了电池使用寿 命; 另一方面, 纳米 $\mathrm{Zn}$ 的比表面积较高, 增加了与电解 液的接触面积(图 S13). 图 $5 \mathrm{~A}$ 为准固态 ZIBs 在 0.5 $\mathrm{mA} / \mathrm{cm}^{2}$ 电流密度下的充放电曲线. 如图所示, 该 ZIBs 具有良好的比容量 $\left(109 \mathrm{mAh} / \mathrm{g}, 0.869 \mathrm{mAh} / \mathrm{cm}^{2}\right)$. 为了评 价 ZIBs 的实际应用价值, 将三个准固态 ZIBs 串联制备 成电池组. 结果表明, 该电池组可以有效驱动 $3 \mathrm{~V}$ 的 LED (图 5B, 5C 和图 S14).

此外, 所制备的 ZIBs 在 $0.5 \mathrm{~mA} / \mathrm{cm}^{2}$ 电流密度下循 环 200 圈后容量保持率维持在 $90 \%$ 以上(图 5D), 表明该 ZIBs 具有优异的电化学循环稳定性. 这不仅取决于 $\mathrm{BC} / \mathrm{PANI} / \mathrm{CNTs}$ 柔性正极, 而 $\mathrm{Zn} / \mathrm{CC}$ 负极的设计同样重 要. 目前, ZIBs 的研究多采用以商业 $\mathrm{Zn}$ 箔作为负极. 但 是 $\mathrm{Zn}$ 箔在水系电解液中枝晶问题严重, 这极大地影响 了 ZIBs 的循环寿命和电池容量. 此外, 以远远过量的 $\mathrm{Zn}$ 作为负极也降低了 $\mathrm{Zn}$ 的利用率, 增加了使用成本. 当前, 在基底上沉积 $\mathrm{Zn}$ 是提高 $\mathrm{Zn}$ 负极循环寿命的有效 方案之一, 常见的基底有石墨纸[7]和包覆导电浆料的纤 维素纱线 ${ }^{[42]}$ 等. 因此, 我们在兼具高机械强度和导电性 的 $\mathrm{CC}$ 基底上负载 $\mathrm{Zn}$ 用作负极, 不仅增强了 $\mathrm{Zn}$ 的稳定 性、保证了电池的长循环稳定性, 同时也提高了 $\mathrm{Zn}$ 的 利用率. 我们进一步通过测试准固态 ZIBs 在不同弯曲 次数后的放电特性来分析该器件的形变稳定性. 如图 $\mathrm{S} 15$ 所示, 该准固态 ZIBs 在 $1 \mathrm{~mA} / \mathrm{cm}^{2}$ 的电流密度下, 弯 曲 200 次后, 容量保持率仍接近 $70 \%$.
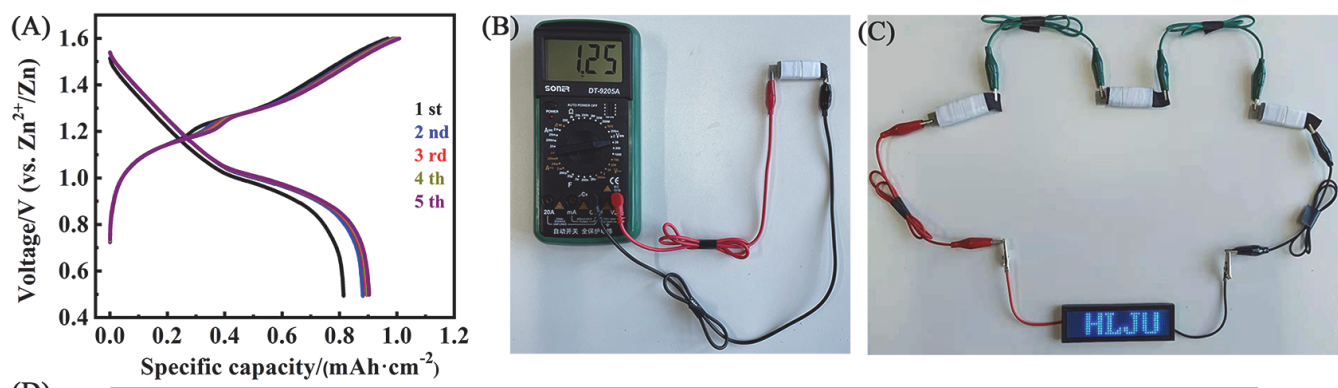

(D)

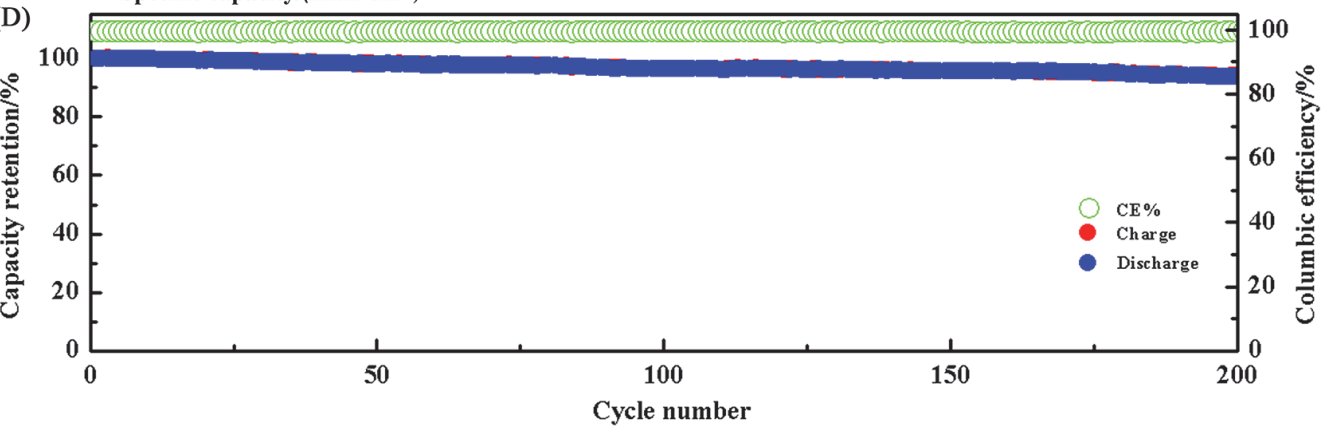

图 5 (A) 准固态 ZIBs 在 $0.5 \mathrm{~mA} / \mathrm{cm}^{2}$ 时的充放电曲线; (B) ZIBs 开路电压的照片; (C) 三个 ZIBs 串联驱动 $3 \mathrm{~V} \mathrm{LEDs}$ 的照片; (D) ZIBs 在 $0.5 \mathrm{~mA} / \mathrm{cm}^{2}$ 下的循环稳定性

Figure 5 (A) Charge-discharge curves of the quasi-solid-state ZIBs at $0.5 \mathrm{~mA} / \mathrm{cm}^{2}$; (B) open circuit voltage of the ZIBs; (C) three ZIBs connected in series to power $3 \mathrm{~V}$ LEDs; (D) cycling performance of the ZIBs at $0.5 \mathrm{~mA} / \mathrm{cm}^{2}$ 


\section{3 结论}

总之, 我们采用简便的方法制备了用于柔性 ZIBs 的 $\mathrm{BC} / \mathrm{PANI} / \mathrm{CNTs}$ 正极. $\mathrm{BC}$ 作为基底材料在构建电极结 构及提高电极的力学特性方面显示出巨大的潜力. 此 外, BC 基底、 PANI 活性物质和 CNTs 高导电材料间的 协同作用有助于柔性电极整体性能的提升. 以 BC/PANI/CNTs 柔性电极装配的 ZIBs 获得了 $157 \mathrm{mAh} / \mathrm{g}$ $\left(1.148 \mathrm{mAh} / \mathrm{cm}^{2}\right)$ 的高比容量, 且经过 1000 次充放电循 环后, 容量保持率可维持在 $50 \%$ 以上, 表现出良好的循 环稳定性. 组装的准固态 ZIBs 在 $0.5 \mathrm{~mA} / \mathrm{cm}^{2}$ 电流密度 时表现出 $109 \mathrm{mAh} / \mathrm{g}\left(0.869 \mathrm{mAh} / \mathrm{cm}^{2}\right)$ 的比容量. 此外, 该准固态 ZIBs 在 $0.5 \mathrm{~mA} / \mathrm{cm}^{2}$ 的低电流密度下, 经 200 圈充放电循环后, 仍获得了高于 $90 \%$ 的容量保持率. 三 个 ZIBs 串联后可以驱动 $3 \mathrm{~V}$ 的 LEDs. 这项工作提供了 一种简单、低成本的方法制备具有高电化学性能及高柔 韧性的柔性电极, 为柔性 ZIBs 的发展提供了新思路.

\section{4 实验部分}

\subsection{BC/PANI 电极的制备}

(1) BC 悬浮液: 以海南益德食品有限公司的颗粒状 $\mathrm{BC}$ 为原材料, 用去离子水将其反复洗涤, 直至 $\mathrm{pH}$ 值为 中性. 然后采用高速匀浆机以 $10000 \mathrm{r} / \mathrm{min}$ 的搅拌速度 将一定量的 $\mathrm{BC}$ 颗粒切碎, 获得均匀的 $\mathrm{BC}$ 悬浮液. 最后 取 $10 \mathrm{~mL} \mathrm{BC}$ 悬浮液干燥, 通过称取干燥前后的质量差, 计算出 $\mathrm{BC}$ 悬浮液的准确浓度.

(2) BC/PANI 电极: 采用原位氧化聚合的方法在 BC 纤维表面生长 PANI 活性材料. 首先将 $13.30 \mathrm{~mL}$ 浓度为 $1.87 \mathrm{mg} / \mathrm{mL}$ 的 BC 悬浮液均匀分散在 $10 \mathrm{~mL} 2 \mathrm{~mol} / \mathrm{L} \mathrm{HCl}$ 溶液中. 然后在上述混合液中加入 $20 \mathrm{~mL}$ 含有 $0.07 \mathrm{~mL}$ 苯胺单体(AN)的 $2 \mathrm{~mol} / \mathrm{L} \mathrm{HCl}$ 溶液, 并在冰水浴中搅拌 $30 \mathrm{~min}$. 另取 $0.2826 \mathrm{~g} \mathrm{APS}$ 溶于 $10 \mathrm{~mL} 2 \mathrm{~mol} / \mathrm{L} \mathrm{HCl}$ 溶液 中, 之后在冰水浴 $0 \sim 5{ }^{\circ} \mathrm{C}$ 条件下将其逐滴加入上述混 合液进行反应, 反应时间为 $10 \mathrm{~h}$. 待反应完成后, 将产 物通过真空过滤成膜, 并用去离子水反复冲洗. 随后将 膜置于 $60{ }^{\circ} \mathrm{C}$ 烘箱中干燥 $6 \mathrm{~h}$, 制得产物命名为 BC/PANI (或 BC/PANI-0.07). 当添加的 $\mathrm{AN}$ 为 0.06 和 $0.08 \mathrm{~mL}$ 时, 所得产物命名为 $\mathrm{BC} / \mathrm{PANI}-0.06$ 和 $\mathrm{BC} / \mathrm{PANI}-0.08$. BC/PANI-0.06、BC/PANI 和 BC/PANI-0.08 中活性物质 PANI 的单位面积负载量分别为 $6.50 、 7.69$ 和 10.00 $\mathrm{mg} / \mathrm{cm}^{2}$.

\subsection{BC/PANI/CNTs 柔性电极的制备}

根据上述步骤, 分别在 $\mathrm{BC} / \mathrm{PANI}$ 反应液中加入不 同量(5、10、15 mg)酸化处理的 CNTs, 然后对整个分散 液进行真空过滤. 得到的三种膜材料经去离子水反复冲 洗后, 在烘箱中于 $60{ }^{\circ} \mathrm{C}$ 条件下干燥 $6 \mathrm{~h}$, 最终产物分别 命名为 $\mathrm{BC} / \mathrm{PANI} / \mathrm{CNTs}-5 、 \mathrm{BC} / \mathrm{PANI} / \mathrm{CNTs}$ (或 BC/PANI/ CNTs-10)、BC/PANI/CNTs-15. 各电极中活性物质 PANI
的负载量分别为 $7.35 、 7.30$ 和 $7.31 \mathrm{mg} / \mathrm{cm}^{2}$. 对比样品 PANI/CNTs 的制备工艺除了不加入 BC, 其余步骤同上.

\section{$4.3 \mathrm{Zn} / \mathrm{CC}$ 负极的制备}

采用 CHI760E 电化学工作站, 通过电化学沉积的 方法, 在 $\mathrm{CC}$ 上负载 $\mathrm{Zn}$, 制备 $\mathrm{Zn} / \mathrm{CC}$ 负极. 电化学沉积 采用两电极装置, 其中锌片作为工作电极, $\mathrm{CC}$ 作为对电 极及参比电极, $1 \mathrm{~mol} / \mathrm{L} \mathrm{ZnSO}_{4}$ 为电解液. 沉积电位为 $0.8 \mathrm{~V}$, 沉积时间为 $600 \mathrm{~s}$.

\subsection{ZIBs 电池的制备}

（1）基于水系电解液的 ZIBs: 正极为 BC/PANI/ $\mathrm{CNTs}$ 电极, 负极为 $\mathrm{Zn}$ 馢, 隔膜为聚酰胺膜, 电解液为 2 $\mathrm{mol} / \mathrm{L} \mathrm{ZnSO}_{4}$ 水溶液.

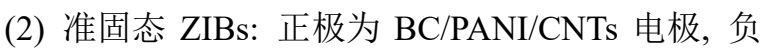
极为 $\mathrm{Zn} / \mathrm{CC}$, 凝胶电解质为 $\mathrm{PVA} / \mathrm{ZnSO}_{4}$. 其中, $\mathrm{PVA} / \mathrm{ZnSO}_{4}$ 的制备工艺如下, 将 $1 \mathrm{~g} \mathrm{PVA}$ 溶解在 $10 \mathrm{~mL}$ 的 $2 \mathrm{~mol} / \mathrm{L} \mathrm{ZnSO}_{4}$ 中, 然后搅拌加热到 $90{ }^{\circ} \mathrm{C}$, 直到溶液 清澈, 最后室温冷却.

\section{5 材料表征}

扫描电子显微镜选用 Hitachi S-4800; X 射线衍射仪 选用 RigakuD/max-IIIB, 测试条件为 $\mathrm{Cu} \mathrm{K \alpha}$ 辐射 $(\lambda=$ $0.15406 \mathrm{~nm})$, 扫描范围 $(2 \theta)$ 为 $10^{\circ} \sim 80^{\circ} ; \mathrm{X}$ 射线光电子 能谱仪选用 VG ESCALAB MKII; 拉曼光谱仪选用 JobinYvonHR800; 傅里叶红外光谱仪选用 Thermo Nicolet iS50 FT-IR.

\section{6 电化学表征}

采用 Autolab 电化学工作站分析电极及 ZIBs 的循环 伏安特性; 采用 Neware 电池测试系统分析电极及 ZIBs 的充放电特性; 采用 Autolab 电化学工作站分析电极的 电化学阻抗, 频率范围为 $0.001 \mathrm{~Hz} \sim 100 \mathrm{kHz}$, 振幅为 5 $\mathrm{mV}$.

\section{References}

[1] Lei, H.; Wang, Z. L.; Yang, F.; Huang, X. Q.; Liu, J. H.; Liang, Y. Y.; Xie, J. P.; Javed, M. S.; Lu, X. H.; Tan, S. Z.; Mai, W. J. Nano Energy 2020, 68, 104293.

[2] Li, H. F.; Han, C. P.; Huang, Y.; Huang, Y.; Zhu, M. S.; Pei, Z. X.; Xue, Q.; Wang, Z. F.; Liu, Z. X.; Tang, Z. J.; Wang, Y. K.; Kang, F. Y.; Li, B. H.; Zhi, C. Y. Energy Environ. Sci. 2018, 11, 941

[3] Liao, M.; Wang, J.; Ye, L.; Sun, H.; Wen, Y.; Wang, C.; Sun, X.; Wang, B.; Peng, H. Angew. Chem., Int. Ed. 2020, 59, 2273.

[4] Liang, H.; Cao, Z.; Ming, F.; Zhang, W.; Anjum, D. H.; Cui, Y.; Cavallo, L.; Alshareef, H. N. Nano Lett. 2019, 19, 3199.

[5] Li, Y.-L.; Yu, D.-D.; Lin, S.; Sun, D.-F.; Lei, Z.-Q. Acta Chim. Sinica 2021, 79, 200 (in Chinese). (李燕丽, 于丹丹, 林森, 孙东飞, 雷自 强, 化学学报, 2021, 79, 200.)

[6] Wu, F.; Gao, X.; Xu, X.; Jiang, Y.; Gao, X.; Yin, R.; Shi, W.; Liu, W.; Lu, G.; Cao, X. ChemSusChem 2020, 13, 1537.

[7] Ma, Y.; Xie, X.; Lv, R.; Na, B.; Ouyang, J.; Liu, H. ACS Sustainable Chem. Eng. 2018, 6, 8697.

[8] Liu, J.-T.; Xie, Y.; Gao, Q.; Cao, F.-H.; Qin, L.; Wu, Z.-Y.; Zhang, W.; Li, H.; Zhang, C.-L. Eur. J. Inorg. Chem. 2020, $2020,581$.

[9] Cong, H.-P.; Ren, X.-C.; Wang, P.; Yu, S.-H. Energy Environ. Sci. 2013, 6, 1185 .

[10] Yano, H.; Sugiyama, J.; Nakagaito, A. N.; Nogi, M.; Matsuura, T.; 
Hikita, M.; Handa, K. Adv. Mater. 2005, 17, 153.

[11] Hu, W.; Chen, S.; Xu, Q.; Wang, H. Carbohydr. Polym. 2011, 83, 1575.

[12] Zhang, X.; He, M.; He, P.; Li, C.; Liu, H.; Zhang, X.; Ma, Y. Appl. Surf. Sci. 2018, 433, 419.

[13] Li, S.; Huang, D.; Zhang, B.; Xu, X.; Wang, M.; Yang, G.; Shen, Y. Adv. Energy Mater. 2014, 4, 1301655

[14] Wang, Y.; Wang, X.; Li, X.; Bai, Y.; Xiao, H.; Liu, Y.; Liu, R.; Yuan, G. Adv. Funct. Mater. 2019, 29, 1900326.

[15] Liu, R.; Ma, L.; Niu, G.; Li, X.; Li, E.; Bai, Y.; Yuan, G. Adv. Funct. Mater. 2017, 27, 1701635.

[16] Ma, L.; Liu, R.; Niu, H.; Xing, L.; Liu, L.; Huang, Y. ACS Appl. Mater. Interfaces 2016, 8, 33608 .

[17] Wang, Y.; Wang, X.; Li, X.; Bai, Y.; Xiao, H.; Liu, Y.; Liu, R.; Yuan, G. Adv. Funct. Mater. 2019, 29, 1900326.

[18] Kim, C.; Ahn, B. Y.; Wei, T. S.; Jo, Y.; Jeong, S.; Choi, Y.; Kim, I. D.; Lewis, J. A. ACS Nano 2018, 12, 11838

[19] Cao, H.; Wan, F.; Zhang, L.; Dai, X.; Huang, S.; Liu, L.; Niu, Z. J. Mater. Chem. A 2019, 7, 11734.

[20] Li, X.; Lv, R.; Zou, S.; Na, B.; Liu, P.; Ma, Y.; Liu, H. Compos. Sci. Technol. 2019, 180, 71 .

[21] Zhang, Y.; Wang, Q.; Bi, S.; Yao, M.; Wan, F.; Niu, Z. Nanoscale 2019, 11, 17630 .

[22] Shi, H. Y.; Ye, Y. J.; Liu, K.; Song, Y.; Sun, X. Angew. Chem., Int. Ed. 2018, 57, 16359.

[23] Wan, F.; Zhang, L.; Wang, X.; Bi, S.; Niu, Z.; Chen, J. Adv. Funct. Mater. 2018, 28, 1804975.

[24] Han, J.; Wang, K.; Liu, W.; Li, C.; Sun, X.; Zhang, X.; An, Y.; Yi, S.; Ma, Y. Nanoscale 2018, 10, 13083.

[25] Gao, Z.-Z.; Tong, H.; Chen, J.-H.; Yue, S.-H.; Bai, W.-L.; Zhang, X.-G.; Pan, Y.-F.; Shi, M.; Song, Y.-X. Acta Chim. Sinica 2014, 72, 1175 (in Chinese). (高珍珍, 佟浩, 陈建慧, 岳世鸿, 白文龙, 张 校刚，潘燕飞，石明，宋玉翔，化学学报, 2014, 72, 1175.)
[26] Jia, W.; Xu, M.-W.; Lei, C.; Bao, S.-J.; Jia, D.-Z. Acta Chim. Sinica 2011，69, 1773 (in Chinese). (贾巍, 徐茂文, 雷超, 包淑娟, 贾殿 赠, 化学学报, 2011, 69, 1773.)

[27] Kang, Y. J.; Chun, S. J.; Lee, S. S.; Kim, B. Y.; Kim, J. H.; Chung, H.; Lee, S. Y.; Kim, W. ACS Nano 2012, 6, 6400.

[28] Wang, H.; Zhu, E.; Yang, J.; Zhou, P.; Sun, D.; Tang, W. J. Phys Chem. C 2012, 116, 13013.

[29] Liu, M.; Miao, Y. E.; Zhang, C.; Tjiu, W. W.; Yang, Z.; Peng, H.; Liu, T. Nanoscale 2013, 5, 7312.

[30] Meng, Y.; Wang, K.; Zhang, Y.; Wei, Z. Adv. Mater. 2013, 25, 6985.

[31] Rashidi, M.; Tavasoli, A. J. Supercrit. Fluid. 2015, 98, 111.

[32] Chen, M. L.; Oh, W. C. Nanoscale Res. Lett. 2011, 6, 398.

[33] Hu, W.; Chen, S.; Yang, Z.; Liu, L.; Wang, H. J. Phys. Chem. B 2011, 115, 8453 .

[34] Mo, Z.-L.; Zhao, Z.-L.; Chen, H.; Niu, G.-P.; Shi, H.-F. Carbohydr. Polym. 2009, 75, 660 .

[35] Cong, H.-P.; Ren, X.-C.; Wang, P.; Yu, S.-H. Energy Environ. Sci. 2013, 6,1185 .

[36] Kumar, A.; Kumar, V.; Kumar, M.; Awasthi, K. Polym. Compos. 2018, 39, 3858 .

[37] Li, S.; Huang, D.; Zhang, B.; Xu, X.; Wang, M.; Yang, G.; Shen, Y. Adv. Energy Mater. 2014, 4, 1301655.

[38] Wu, J.; Zhang, Q. e.; Wang, J.; Huang, X.; Bai, H. Energy Environ. Sci. 2018, 11, 1280.

[39] Han, J.; Wang, K.; Liu, W.; Li, C.; Sun, X.; Zhang, X.; An, Y.; Yi, S.; Ma, Y. Nanoscale 2018, 10, 13083.

[40] Zang, X.; Li, X.; Zhu, M.; Li, X.; Zhen, Z.; He, Y.; Wang, K.; Wei, J.; Kang, F.; Zhu, H. Nanoscale 2015, 7, 7318.

[41] Yu, P.; Zhang, Z.; Zheng, L.; Teng, F.; Hu, L.; Fang, X. Adv. Energy Mater. 2016, 6, 1601111.

[42] Yi, H.; Ma, Y.; Zhang, S.; Na, B.; Zeng, R.; Zhang, Y.; Lin, C. ACS Sustainable Chem. Eng. 2019, 7, 18894.

(Cheng, B.) 